\title{
Distinguishing Features: Staphylococcal Scalded Skin Syndrome vs. Toxic Epidermal Necrolysis
}

\author{
Hannah Badon BS ${ }^{a}$, Joy King MD', Robert T. Brodell MD ${ }^{b, c}$, Adam Byrd MD ${ }^{d}$ \\ aUniversity of Mississippi Medical Center, Jackson, MS \\ ${ }^{\mathrm{b}}$ Department of Pathology, University of Mississippi Medical Center, Jackson, MS \\ ${ }^{\mathrm{C} D e p a r t m e n t}$ of Dermatology, University of Mississippi Medical Center, Jackson, MS
}

\section{ABSTRACT}

Staphylococcal scalded skin syndrome (SSSS) and toxic epidermal necrolysis (TEN) are dermatologic conditions that have a similar clinical appearance. Careful attention to clinical features, such as the coloration at the base of the blister, and histopathology are utilized to make an accurate diagnosis. While supportive therapy is required for both conditions, SSSS requires appropriate antibiotics to treat the underlying staphylococcus and TEN requires elimination of an offending drug (usually an antibiotic).

\section{INTRODUCTION}

Staphylococcal scalded skin syndrome (SSSS) and toxic epidermal necrolysis (TEN) share many clinical features. Both demonstrate widespread blistering, positive Nikolsky sign and tender skin. In fact, when TEN was first described in 1956, it was reported to be "an eruption resembling scalding of the skin." There are many differences, however. Staphylococcal scalded skin syndrome is usually seen in young children and infants, though it can be seen in adults with renal failure. The mortality of TEN $(25 \%-35 \%)^{2}$ is generally higher than SSSS (4\% in children and infants). However, due to comorbid conditions, the mortality of SSSS in adults can be as high as $60 \% .^{3}$ Most importantly, these conditions have entirely different pathophysiologic mechanisms.
Distinguishing between these two conditions with similar clinical features early in the course of the process is important since the correct diagnosis impacts both the prognosis and treatment approach.

\section{PATHOPHYSIOLOGY}

There are notable differences in the pathophysiologic basis of these two conditions (Table 1). Histologically, in SSSS, the epidermis is detached from the granular layer and blisters are subcorneal in location so that the skin turns red and "slides off in sheets." ${ }^{4}$ This detachment occurs due to the bacteria and its exotoxin, a serine protease that destroys desmoglein $1 .^{5}$ The exfoliative toxins $A$ and $B$ are the specific 
exotoxins that cause this detachment and are only produced by approximately $5 \%$ of Staphylococcal aureus bacteria. ${ }^{5}$ These are the same toxins that produce bullous impetigo. Widespread blistering only occurs in infants with immature kidneys and adults with renal failure who cannot clear the toxins rapidly. Because the blistering is superficial, it does not cause scarring. The poor prognosis in adults with SSSS is due to the severity of the underlying Staphylococcal infection in addition to that of the significant renal impairment. It is not uncommon for adults over the age of 70 to experience chronic renal disease, and this pathological process can result in failure to excrete exotoxins produced by staphylococcal bacteria. ${ }^{5}$

TEN is a complication associated with a newly administered medication. While it is rare, it is accompanied by a mortality rate between $25 \%$ and $35 \%$ due to the extensive nature of the dermal-epidermal blistering process which is similar in its impact to a $3^{\text {rd }}$ degree burn. ${ }^{2}$ The pathophysiological mechanism of TEN is largely unknown, but current studies believe that it is due to a delayed hypersensitivity reaction along with a genetic component involving one's abilities to destroy drug metabolites. The necrosis factor of TEN is mediated by CD8 cells that induce apoptosis of keratinocytes. ${ }^{6}$

Table 1. Comparison of salient features of staphylococcal scalded skin syndrome and toxic epidermal necrolysis

$$
\text { Staphylococcal Scalded Toxic Epidermal Necrolysis }
$$

Skin Syndrome

\begin{tabular}{lll}
\hline Blister coloration & Tan or Brown & White \\
$\begin{array}{l}\text { Mucosa involvement } \\
\text { Affected skin layer }\end{array}$ & No & Yes \\
& Subcorneal (desmoglein 1) & $\begin{array}{l}\text { Epidermal-dermal junction } \\
\text { (keratinocytes) }\end{array}$ \\
\hline Mortality & Greater mortality in adults & Greater mortality in children \\
\hline
\end{tabular}

\section{DISTINGUISHING FEATURES}

The simplest way to distinguish SSSS and TEN in Fitzpatrick skin types IV-VI is to examine the coloration at the base of a blister compared to normal skin. The superficial blisters of SSSS show the same color at the base of the blister as adjacent skin (Figure 1). The dermal-epidermal blister of TEN demonstrates a white or pink basethe color of the dermis-depending upon the amount of vasculature, while surrounding skin is tan, brown, or black due to melanin pigment at and above the basal layer of the epidermis (Figure 2). ${ }^{7}$
Routine histological examination is the gold standard for distinguishing SSSS from TEN. SSSS demonstrates a subcorneal blister usually with scant inflammation (Figure 3). TEN shows a dermal-epidermal blister with focal dyskeratosis and areas of full thickness epidermal necrosis (Figure 4). Unfortunately, this approach can delay the diagnosis by 48 hours due to the processing time for standard hematoxylin and eosin sections. 
Frozen section histologic technology permits a faster assessment of the location of the blister, but the degree of dyskeratosis and

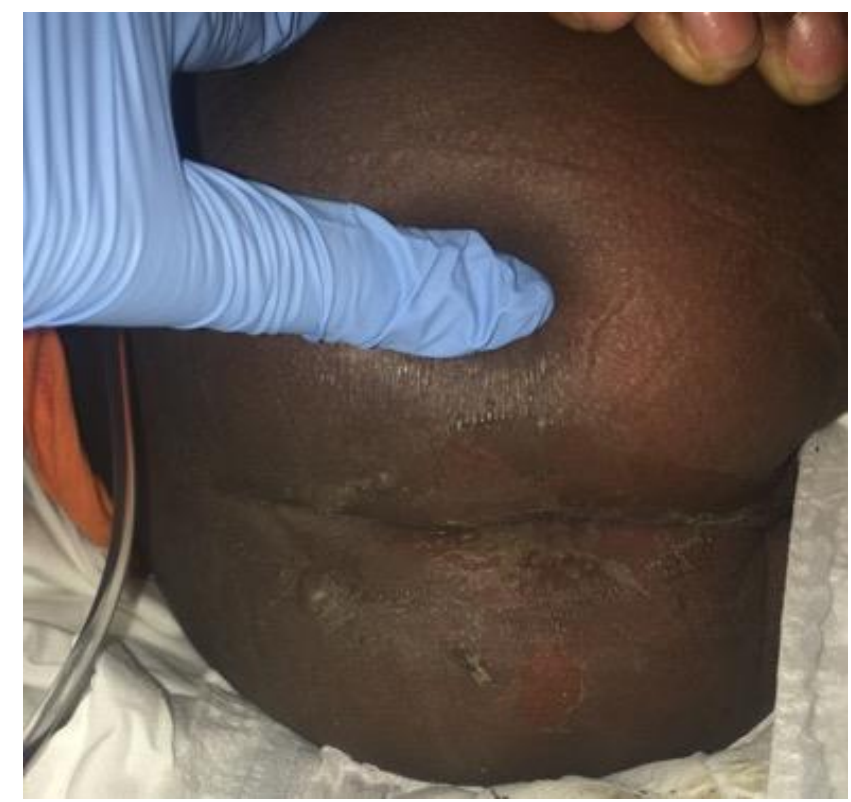

Figure 1. The base of a blister in a patient with Staphylococcal Scalded Skin Syndrome demonstrating the brown base resulting from a sub-corneal blistering process.

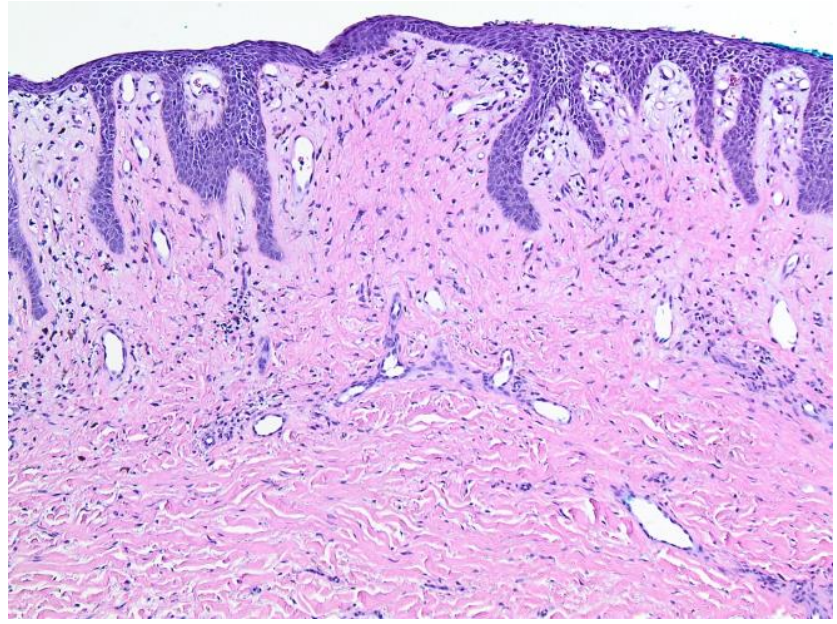

Figure 3. SSSS—Subcorneal splitting (granular layer) of epidermis with intracellular spongiosis. Sparse interstitial lymphohistiocytic infiltrate and telangiectasias in the dermis. No epidermal necrosis is noted (H\&E, 100X). Provided by Dr. Joy King. the nature of the underlying inflammation is harder to assess when compared to permanent sections.

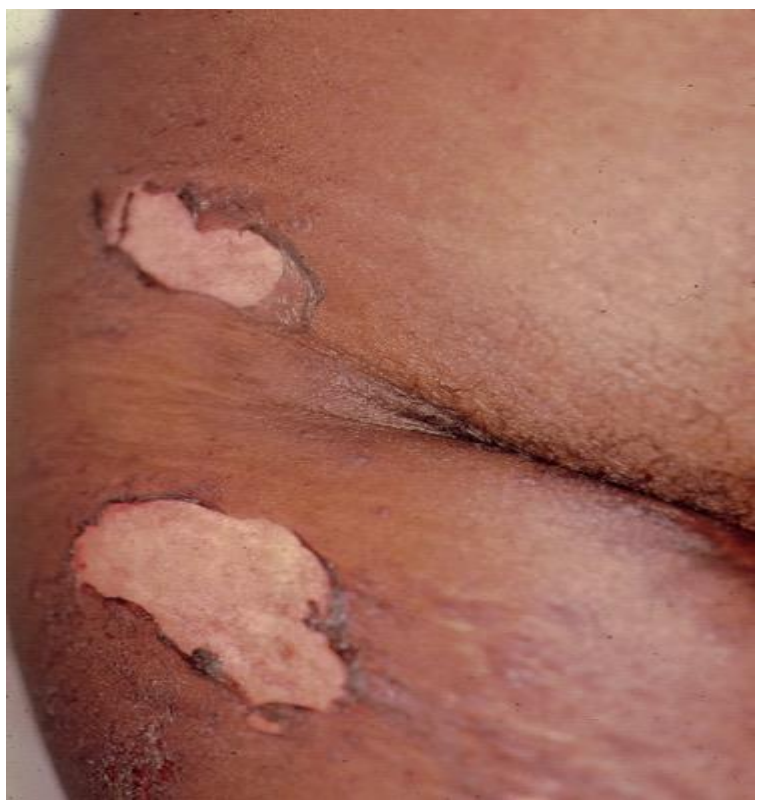

Figure 2. The base of a blister in a patient with toxic epidermal necrolysis showing the white coloration of the dermis exposed with dermal-epidermal blistering.

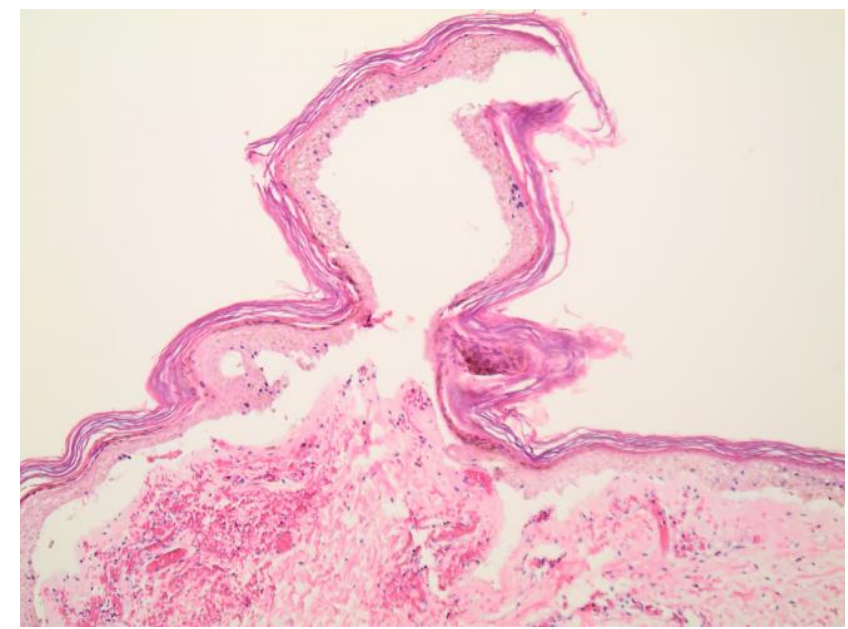

Figure 4. TEN—Full-thickness epidermal necrosis and underlying dermal-epidermal separation with sparse dermal inflammatory infiltrate and marked red blood cell extravasation. (H\&E, 100X). Provided by Dr. Joy King. 


\section{TREATMENT}

Treatment for SSSS generally involves supportive therapy with the addition of appropriate antibiotics to eradicate the underlying Staphylococcal infection. Most SSSS causing strains of Staphylococcal aureus are resistant to penicillin, but synthetic substitutes such as nafcillin or oxacillin are usually successful.

Additionally, SSSS can be caused by Methicillin-resistant (MRSA) bacteria, so antibiotics such as Vancomycin should be utilized if MRSA is suspected. ${ }^{7}$ Infants that have a higher chance of developing a metabolic imbalance even with superficial blistering may require supportive therapy including intravenous Ringer's solution to replenish fluid loss; however, dermal substitutes such as Omniderm (C) and Suprathel $@$ may be necessary. ${ }^{8}$

The first step in the treatment of toxic epidermal necrolysis is to discontinue the offending medication. Supportive care in a burn unit utilizing intravenous fluids is imperative to maintain metabolic equilibrium and avoid fluid-balance complications that can lead to death. Topical antiseptics such as silver sulfadiazine (Silvadine(C) are commonly used along with non-adherent gauze to cover the blistering areas. Appropriate antibiotics can be given in response to secondary infections. Evolving systemic treatments for TEN include short courses of glucocorticosteroids, cyclosporine, intravenous immunoglobulins, and anti-TNF biologic therapy. Further studies should be conducted to delineate the position of each of these options. ${ }^{9}$
Conflict of Interest Disclosures: Dr. Brodell has participated in multi-center clinical trials for Galderma Laboratories, L.P., Novartis, and GlaxoSmithKline. He serves on the editorial boards of the American Medical Student Research Journal, Practice Update Dermatology, Practical Dermatology, Journal of the Mississippi State Medical Society, and SKIN: The Journal of Cutaneous Medicine.

Funding: None.

Corresponding Author:

Robert T. Brodell, MD

Department of Dermatology

University of Mississippi Medical Center

2500 North State Street, Jackson, MS

601-815-8000 (Office)

602-984-2250 (Fax)

rbrodell@umc.edu
References:

1. Lyell A. Toxic epidermal necrolysis: an eruption resembling scalding of the skin. Br J Dermatol. 1956; 68: 355-361.

2. Miliszewski MA, Kirchhof MG, Sikora S, et al. Stevens-Johnson syndrome and toxic epidermal necrolysis: an analysis of triggers and implications for improving prevention. Am J Med. 2016; 129: 12211225 .

3. Patel GK, Finlay AY. Staphylococcal scalded skin syndrome: diagnosis and management. Am J Clin Dermatol. 2003; 4: $165-175$. 
4. Melish ME, Glasgow LA, Turner

MD. The staphylococcal scalded-skin

syndrome: isolation and partial

characterization of the exfoliative

toxin. J Infect Dis. 1972; 125: 129-140.

5. Handler MZ, Schwartz RA.

Staphylococcal scalded skin syndrome:

diagnosis and management in children

and adults. $J$ Eur Acad Dermatol

Venereol. 2014; 28: 1418-1423.

6. Wong A, Malvestiti AA, Hafner MF.

Stevens-Johnson syndrome and toxic epidermal necrolysis: a review. Revista da Associacao Medica Brasileira 1992; 62: 468-473.

7. Mishra AK, Yadav P, Mishra A. A systemic review on Staphyloccal scalded skin syndrome (SSSS): a rare and critical disease of neonates. Open Microbiol J. 2016; 31: 150-159.

8. Baartmans MG, Dokter J, den Hollander JC, et al. Use of skin substitute dressings in the treatment of staphylococcal scalded skin syndrome in neonates and young infants. Neonatology 2011; 100: 9-13.

9. Paulman M, Mockenhaupt M. Fever in Stevens-Johnson syndrome and toxic epidermal necrolysis in pediatric cases: laboratory work-up and antibiotic therapy. Pediatri Infect Dis J. 2017; 36: 513-515. 